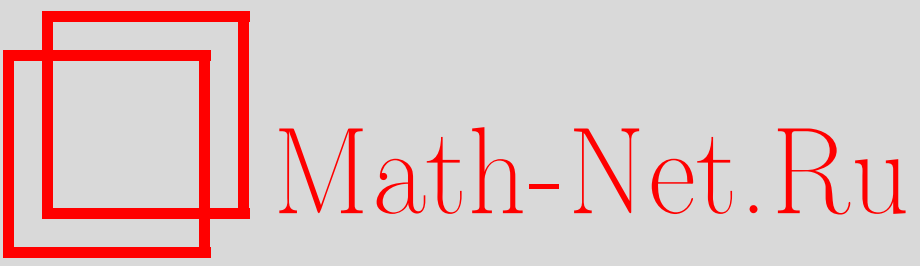

У. А. Розиков, Построение несчетного числа предельных гиббсовских мер неоднородной модели Изинга, ТМФ, 1999, том 118, номер 1, 95-104

DOI: https://doi.org/10.4213/tmf688

Использование Общероссийского математического портала Math-Net.Ru подразумевает, что вы прочитали и согласны с пользовательским соглашением

http://www.mathnet.ru/rus/agreement

Параметры загрузки:

IP: 52.205.19.152

26 апреля 2023 г., 10:05:05 
ТЕОРЕТИЧЕСКАЯ

И МАТЕМАТИЧЕСКАЯ

ФИЗИКА

Том 118, № 1

январь, 1999

(C) 1999 г.

У. А. Розиков*

\section{ПОСТРОЕНИЕ НЕСЧЕТНОГО ЧИСЛА ПРЕДЕЛЬНЫХ ГИББСОВСКИХ МЕР НЕОДНОРОДНОЙ МОДЕЛИ ИЗИНГА}

Для неоднородной модели Изинга на дереве Кэли доказано, что существует несчетное число предельных гиббсовских мер, и дано их конструктивное описание.

Данная работа является продолжением недавно опубликованной работы [1].

Пусть $\Gamma^{k}=(V, L)$ - дерево Кэли порядка $k \geqslant 1$, где $V$ - множество вершин, а $L-$ множество ребер данного дерева $[2,3]$.

Для произвольной точки $x^{0} \in V$ положим

$$
\begin{aligned}
W_{n} & =\left\{x \in V: d\left(x, x^{0}\right)=n\right\}, \\
V_{n} & =\bigcup_{m=0}^{n} W_{m}=\left\{x \in V: d\left(x, x^{0}\right) \leqslant n\right\}, \\
L_{n} & =\left\{\langle x, y\rangle \in L: x, y, \in V_{n}\right\},
\end{aligned}
$$

где $d(x, y)$ - расстояние на $\Gamma^{k}$, т.е. число ребер кратчайшего пути, соединяющего $x$ и $y$. Говорят, что $x<y$, если путь из $x^{0}$ в $y$ проходит через $x$. При этом вершина $y$ называется "прямым потомком" вершины $x$, если $y>x$ и $x, y$ являются ближайшими соседями. Если $S(x)$ - совокупность "прямых потомков" вершины $x$, то для любой вершины $x \in V$, отличной от $x^{0}$, имеем $|S(x)|=k$ и $\left|S\left(x^{0}\right)\right|=k+1$.

Неоднородная модель Изинга определяется формальным гамильтонианом [4]

$$
H(\sigma)=-\sum_{\langle x, y\rangle} J_{x y} \sigma(x) \sigma(y)
$$

где суммирование ведется по всем парам ближайших соседей $\langle x, y\rangle, J_{x y}$ - действительнозначная функция, задаюшая взаимодействие соседей $x$ и $y, \sigma(x) \in\{-1,1\}$ для любого $x \in V$.

${ }^{*}$ Институт математики им. В.И. Романовского АН РУз, Ташкент, Республика Узбекистан. E-mail: nasyr@uwed.freenet.uz 
Пусть $A \subset V$ - конечное подмножество. Обозначим через $\Omega_{A}=\{-1,1\}^{A}$ пространство конфигураций, определенных на множестве $A$. Пусть $h_{x}$ - действительнозначная функция точки $x \in V$. Для каждого $n$ определим меру $\mu_{n}$ на $\Omega_{V_{n}}$, полагая

$$
\mu_{n}\left(\sigma_{n}\right)=Z_{n}^{-1} \exp \left\{-\beta H_{n}\left(\sigma_{n}\right)+\sum_{x \in W_{n}} h_{x} \sigma(x)\right\}
$$

где $\beta=1 / T$ ( $T$ - температура $), \sigma_{n}=\left\{\sigma(x), x \in V_{n}\right\} \in \Omega_{V_{n}}, Z_{n}$ - нормирующий множитель и

$$
H_{n}\left(\sigma_{n}\right)=-\sum_{\langle x, y\rangle \in L_{n}} J_{x y} \sigma(x) \sigma(y)
$$

Условия согласования для $\mu_{n}\left(\sigma_{n}\right), n \geqslant 1$, определяются равенством

$$
\sum_{\sigma^{(n)}} \mu_{n}\left(\sigma_{n-1}, \sigma^{(n)}\right)=\mu_{n-1}\left(\sigma_{n-1}\right)
$$

где $\sigma^{(n)}=\left\{\sigma(x), x \in W_{n}\right\}$. В этом случае в силу теоремы Колмогорова о продолжении мер существует и притом единственное распределение Гиббса $\mu$ на $\Omega_{V}$.

Теорема 1 [5]. Для того чтобъ мерь (2) удовлетворяли условию (3), необходимо и достаточно, чтобь для $h_{x}, x \in V$, виполнялось равенство

$$
h_{x}=\sum_{y \in S(x)} f\left(h_{y} ; \theta_{x y}\right)
$$

для любого $x \in V$, где $f(h ; \theta)=\operatorname{arcth}(\theta \operatorname{th} h)$ u $\theta_{x y}=\operatorname{th}\left(J_{x y} / T\right)$.

Положим

$$
\mathcal{G}=\left\{\mathbf{h}=\left\{h_{x}, x \in V\right\}: h_{x}=\sum_{y \in S(x)} f\left(h_{y} ; \theta_{x y}\right)\right\} .
$$

В соответствии с теоремой 1 задача описания предельных гиббсовских мер неоднородной модели Изинга сводится к описанию элементов множеств $\mathcal{G}$. Отсюда следует, что при различных совокупностях $\left\{h_{x}, x \in V\right\}$ получаются различные меры.

Пусть $G_{k+1}$ - свободное произведение $k+1$ циклических групп второго порядка с образуюшими $a_{1}, a_{2}, \ldots, a_{k+1}$. Известно, что сушествует взаимно однозначное соответствие между элементами группы $G_{k+1}$ и множеством вершин $V$ дерева Кэли $\Gamma^{k}$ порядка $k \geqslant 1[2]$.

В работах $[1,3]$ построены классы нормальных делителей конечного индекса группы $G_{k+1}$, изучены структуры разбиений на классы смежности группового представления дерева Кэли по нормальным делителям конечного индекса.

Пусть $G_{k+1}$ - правое представление дерева Кэли $\Gamma^{k}$ и $T_{y}(x)=y x ; x, y \in G_{k+1}-$ левый сдвиг на правом представлении $G_{k+1}$. Пусть $H_{0} \subset G_{k+1}$ - нормальный делитель индекса $r[3]$ и $G_{k+1} / H_{0}=\left\{H_{0}, H_{1}, \ldots, H_{r-1}\right\}$ - фактор-группа относительно $H_{0}$. 
ОПРЕДЕЛЕНИЕ. Совокупность чисел $\left\{h_{x}, x \in G_{k+1}\right\}$ называется $H_{0}$-периодической, если для любого $x \in G_{k+1}$ выполняется равенство

$$
h_{x}=h_{T_{y}(x)}, \quad y \in H_{0} .
$$

Мера, соответствуюшая $H_{0}$-периодической совокупности величин $\left\{h_{x}, x \in G_{k+1}\right\}$, называется $H_{0}$-периодической.

В работе [1] для неоднородной модели Изинга описаны периодические распределения Гиббса.

Введем следующие обозначения:

$$
\begin{aligned}
q_{i}(x) & =\left|S(x) \cap H_{i}\right|, \quad i=\overline{0, r-1} ; \\
Q(x) & =\left(q_{0}(x), q_{1}(x), \ldots, q_{r-1}(x)\right) ; \\
q_{i}\left(H_{0}\right) & =q_{i}(e), \quad e-\text { единичный элемент группы } G_{k+1} ; \\
Q\left(H_{0}\right) & =\left(q_{0}\left(H_{0}\right), q_{1}\left(H_{0}\right), \ldots, q_{r-1}\left(H_{0}\right)\right) ; \\
N\left(H_{0}\right) & =\left|\left\{j: q_{j}\left(H_{0}\right) \neq 0\right\}\right|,
\end{aligned}
$$

где $|\cdot|$ - число элементов множества.

Теорема 2 [1]. Для любого $x \in G_{k+1}$ существует перестановка $\pi_{x}$ координат вектора $Q\left(H_{0}\right)$ такая, что

$$
\pi_{x} Q\left(H_{0}\right)=Q(x)
$$

Пусть

$$
N\left(H_{0}\right)=\left|\left\{q_{i_{1}}\left(H_{0}\right), q_{i_{2}}\left(H_{0}\right), \ldots, q_{i_{N\left(H_{0}\right)}}\left(H_{0}\right)\right\}\right|, \quad \theta_{x y}=\theta_{i j}
$$

если $x \in H_{i}, y \in H_{j}$. На параметры $\theta_{i j}$ наложим следуюшее условие.

УСловиЕ I. Пусть для заданных чисел $c_{1}, c_{2}, \ldots, c_{r-1} \in R$ параметры $\theta_{i j}$ удовлетворяют условиям:

1) $c_{\pi_{m}\left(i_{1}\right)} \theta_{m \pi_{m}\left(i_{1}\right)}=c_{\pi_{m}\left(i_{2}\right)} \theta_{m \pi_{m}\left(i_{2}\right)}=\cdots=c_{\pi_{m}\left(i_{N\left(H_{0}\right)}\right)} \theta_{m \pi_{m}\left(i_{N\left(H_{0}\right)}\right)}, m=\overline{0, n-1}$;

2) $\sum_{j=1}^{N\left(H_{0}\right)} q_{i_{j}}\left(H_{0}\right) c_{i_{j}} \theta_{0 i_{j}}-c_{i_{0}} \theta_{0 i_{j_{0}}}>1$;

3) $f\left( \pm h_{0}^{*} ; c_{m}\right)=\sum_{j=1}^{N\left(H_{0}\right)} q_{i_{j}}\left(H_{0}\right) f\left( \pm h_{0}^{*} ; c_{\pi_{m}\left(i_{j}\right)} \theta_{m \pi_{m}\left(i_{j}\right)}\right)-f\left( \pm h_{0}^{*} ; c_{\pi_{m}\left(i_{j_{0}}\right)} \theta_{m \pi_{m}\left(i_{j_{0}}\right)}\right)$, $m=\overline{0, n-1}, j_{0}=\overline{1, N\left(H_{0}\right)}$,

где $\pm h_{0}$ - решения уравнения

$$
h_{0}=\sum_{j=1}^{N\left(H_{0}\right)} q_{i_{j}}\left(H_{0}\right) f\left(h_{0} ; c_{i_{j}} \theta_{0 i_{j}}\right)-f\left(h_{0} ; c_{i_{0}} \theta_{0 i_{j_{0}}}\right), \quad j_{0}=\overline{1, N\left(H_{0}\right)} .
$$

4 Теоретическая и математическая физика, т. 118, № 1, 1999 г. 
В работе [1] при выполнении условия I доказано, что множество $\mathcal{G}$ имеет три $H_{0}$-периодических элемента $\mathbf{h}^{(0)}, \mathbf{h}^{(1)}, \mathbf{h}^{(2)}$, которые имеют вид

$$
\begin{aligned}
& \mathbf{h}^{(0)}=\left\{h_{x}=0 \text { для любого } x \in V\right\}, \\
& \mathbf{h}^{(1)}=\left\{h_{x}=\left\{\begin{array}{ll}
-h_{0}^{*}, & \text { если } x \in H_{0}, \\
-h_{i}^{*}=f\left(-h_{0}^{*} ; c_{i}\right), & \text { если } x \in H_{i}, \quad i=\overline{1, r-1}
\end{array}\right\},\right. \\
& \mathbf{h}^{(2)}=-\mathbf{h}^{(1)},
\end{aligned}
$$

где $c_{i} \in R(i=\overline{0, r-1})$ - заданные числа, $h_{0}^{*}>0$.

Обозначим через $\mu_{0}, \mu_{1}, \mu_{2}$ меры, соответствуюшие совокупностям величин $\mathbf{h}^{(0)}$, $\mathbf{h}^{(1)}, \mathbf{h}^{(2)}$.

В данной работе при выполнении условия I доказывается, что множество $\mathcal{G}$ не меньше несчетного множества, и дается конструктивное описание элементов $\mathcal{G}$. В распределениях, которые мы строим, "1/3-я часть" дерева Кэли занята фазой $\mu_{0}$, " $1 / 3$-я часть" фазой $\mu_{1}$ и " $1 / 3$-я часть" - фазой $\mu_{2}$. Впервые распределения такого типа были построены Добрушиным в модели Изинга на $Z^{3}[6]$. В работах $[3,7-9]$ на дереве Кэли изучены такие распределения, в которых “половина" решетки занята одной фазой, а "половина" - другой фазой рассматриваемых на этой решетке моделей.

Используемый ниже подход основан на теории марковских случайных полей на деревьях и рекуррентных уравнениях этой теории $[1,3,5,7-11]$.

Рассмотрим произвольный (конечный или бесконечный) путь $x^{0}=x_{0}<x_{1}<$ $x_{2}<\cdots$, идущий из точки $x^{0}$. Путь можно изображать последовательностью $i_{1} i_{2} i_{3} \ldots$, где $i_{1}=\overline{0, k} ; \quad i_{n}=\overline{0, k-1}, n \geqslant 2$. Перенумеруем для этого ребра, выходящие вверх из вершины $x \in V$, как $l_{0}(x), l_{1}(x), \ldots, l_{k-1}(x)$ и ребра, выходяшие из вершины $x^{0}$, как $l_{0}\left(x^{0}\right), l_{1}\left(x^{0}\right), \ldots, l_{k-1}\left(x^{0}\right), l_{k}\left(x^{0}\right)$. Тогда пути $x^{0}=x_{0}<x_{1}<x_{2}<\cdots$ можно сопоставить последовательность $i_{1} i_{2} i_{3} \ldots$ такую, что $\left\langle x_{n-1}, x_{n}\right\rangle=l_{i_{n}}\left(x_{n-1}\right), n=1,2, \ldots$, $i_{1}=\overline{0, k} ; \quad i_{n}=\overline{0, k-1}, n \geqslant 2$. Последовательность $i_{1} i_{2} i_{3} \ldots$ однозначно задает путь $x^{0}=x_{0}<x_{1}<x_{2}<\cdots$.

Любой конечный путь $x^{0}=x_{0}<x_{1}<x_{2}<\cdots<x_{n}=x$ длины $n$ определяет точку $x \in W_{n}=\left\{x \in V: d\left(x^{0}, x\right)=n\right\}$. Если путь $x^{0}=x_{0}<x_{1}<x_{2}<\cdots<x_{n}=x$ изображается последовательностью $i_{1} i_{2} \ldots i_{n}$, то мы будем считать, что и вершина $x$ изображается этой последовательностью.

Пусть $x, y \in W_{n}, x$ изображается последовательностью $i_{1} i_{2} \ldots i_{n}$, а $y$ - последовательностью $j_{1} j_{2} \ldots j_{n}$, причем $i_{1}=j_{1}, i_{2}=j_{2}, \ldots, i_{m}=j_{m}$, но $i_{m+1}<j_{m+1}$ для некоторого $m$. В этом случае будем говорить, что $x \prec y$.

Пусть $\pi$ - бесконечный путь, изображаемый последовательностью $i_{1} i_{2} \ldots\left(i_{1}=\overline{0, k}\right.$; $\left.i_{n}=\overline{0, k-1}, n \geqslant 2\right)$. Сопоставим пути $\pi$ вешественное число

$$
t=t(\pi)=\sum_{n=1}^{\infty} \frac{i_{n}}{k^{n}}
$$

Ясно, что $t \in[0 ; 1+1 / k]$. 
Это сопоставление взаимно однозначно всюду, кроме тех чисел $t$, которые разлагаются в конечную сумму

$$
t=\sum_{n=1}^{N} \frac{i_{n}}{k^{n}}, \quad i_{N} \neq 0 .
$$

Обозначим множество таких дробей через $Q_{k}$, т.е.

$$
Q_{k}=\left\{0<t<1+\frac{1}{k}: t=\sum_{n=1}^{N} \frac{i_{n}}{k^{n}}, i_{N} \neq 0\right\} .
$$

Если $t \in Q_{k}$, то число $t$, как известно, можно разложить двумя способами:

$$
\begin{aligned}
t & =\sum_{n=1}^{N} \frac{i_{n}}{k^{n}}, \\
t & =\sum_{n=1}^{N-1} \frac{i_{n}}{k^{n}}+\frac{i_{N}-1}{k^{n}}+\sum_{n=N+1}^{\infty} \frac{k-1}{k^{n}} .
\end{aligned}
$$

Обозначим через $\left\{i_{n}^{(1)}, n=1,2, \ldots\right\}$ первую из этих последовательностей, а через $\left\{i_{n}^{(2)}\right.$, $n=1,2, \ldots\}$ вторую, т.е.

$$
\begin{aligned}
& i_{n}^{(1)}=i_{n}^{(2)}=i_{n}, \quad n \leqslant N-1 ; \\
& i_{N}^{(1)}=i_{N}, \quad i_{n}^{(2)}=i_{N}-1 ; \\
& i_{n}^{(1)}=0, \quad i_{n}^{(2)}=k-1, \quad n \geqslant N+1 .
\end{aligned}
$$

Пусть пути $\pi(t, 1)$ и $\pi(t, 2)$ изображаются соответственно последовательностями $i_{1}^{(1)} i_{2}^{(1)} \ldots$ и $i_{1}^{(2)} i_{2}^{(2)} \ldots$ Ясно, что путь $\pi(t, 1)$ при $n \geqslant N+1$ все время "идет направо", а путь $\pi(t, 2)$ при $n \geqslant N+1$ все время "идет налево".

Пусть $\pi_{1}=\left\{x^{0}=x_{0}<x_{1}<x_{2}<\cdots\right\}$ и $\pi_{2}=\left\{x^{0}=y_{0}<y_{1}<y_{2}<\cdots\right\}$ - бесконечные пути. Сопоставим путям $\pi_{1}, \pi_{2}$ совокупность чисел $\mathbf{h}^{\pi_{1} \pi_{2}}=\left\{h_{x}^{\pi_{1} \pi_{2}}, x \in V\right\}$, удовлетворяющих уравнениям (4).

Предположим, что выполняется условие I. Совокупность $\mathbf{h}^{\pi_{1} \pi_{2}}$ однозначно определяется следуюшими условиями при $x \in W_{n}$ :

$$
h_{x}^{\pi_{1} \pi_{2}}= \begin{cases}-h_{i}^{*}, & \text { если } x \prec x_{n} \text { и } x \in H_{i}, \\ 0, & \text { если } x_{n} \prec x \prec y_{n}, \\ h_{i}^{*}, & \text { если } y_{n} \prec x \text { и } x \in H_{i},\end{cases}
$$

где $h_{i}^{*}, i=\overline{0, r-1}$, определено формулой $(5), n=1,2, \ldots$.

В случае $k=2$ и $H_{0}=\left\{x \in G_{k+1}\right.$ : в несократимой записи $x$ участвуют четные числа $a_{1}$ и $\left.a_{2}\right\}$ вид $\mathbf{h}^{\pi_{1} \pi_{2}}$ на дереве Кэли $\Gamma^{2}$ показан на рисунке. Заметим, что в этом случае индекс нормального делителя $H_{0}$ равен 4 [3].

ТЕОРема 3. Для любых бесконечных путей $\pi_{1}, \pi_{2}$ существует и единственна совокупность чисел $\mathbf{h}^{\pi_{1} \pi_{2}}$, удовлетворяющих соотношениям (4) и (6). 


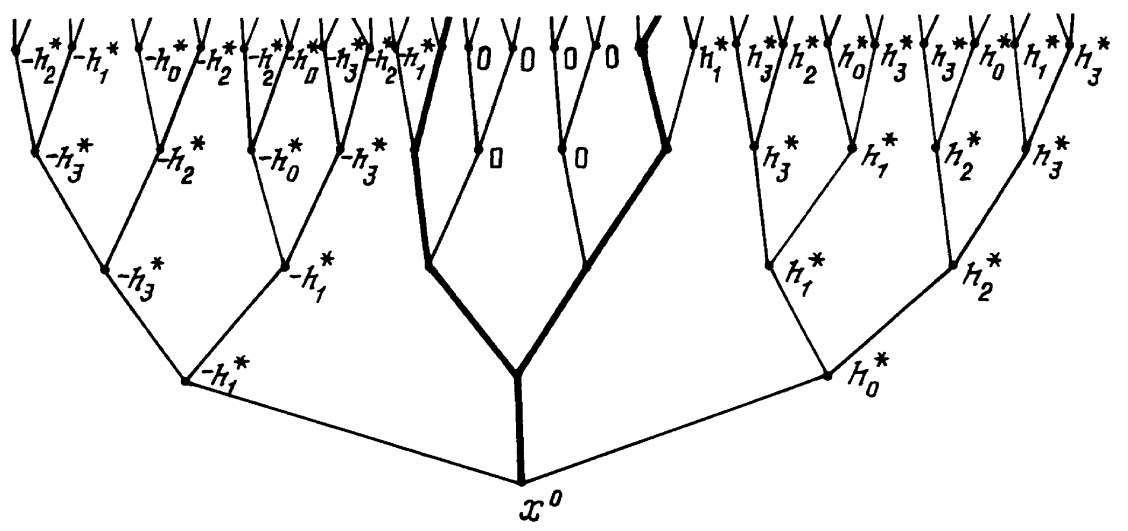

ДокАЗАТЕЛЬСтво. Зададим на множестве $W_{n}$ совокупность

$$
h_{x}^{(n)}= \begin{cases}h_{x}^{\pi_{1} \pi_{2}}, & \text { если } x \in W_{n} \backslash\left\{x_{n}, y_{n}\right\}, \\ h_{x_{n}}^{(n)}, & \text { если } x=x_{n}, \\ h_{y_{n}}^{(n)}, & \text { если } x=y_{n},\end{cases}
$$

где $h_{x}^{\pi_{1} \pi_{2}}$ определяется формулой (6) и $h_{x_{n}}^{(n)}, h_{y_{n}}^{(n)} \in\left[-h_{0}^{*}, h_{0}^{*}\right]$ произвольные. Доопределим с помощью рекуррентных уравнений (4) $h_{x}^{(n)}$ для всех

$$
x \in V_{n}=\bigcup_{m=0}^{n} W_{m} .
$$

Докажем, что предел

$$
h_{x}=\lim _{n \rightarrow \infty} h_{x}^{(n)}
$$

сушествует для любого фиксированного $x \in V$ и этот предел не зависит от выбора $h_{x}^{(n)}$ при $x=x_{n}, y_{n}$.

Так как совокупности чисел $\mathbf{h}^{(0)}, \mathbf{h}^{(1)}, \mathbf{h}^{(2)}$ удовлетворяют равенству (4), заметим, что для любого $m \leqslant n$

$$
h_{x}^{(n)}=\left\{h_{x}^{\pi_{1} \pi_{2}}, x \in W_{n}, x \neq x_{m}, y_{m}\right\} .
$$

Отсюда следует, что при $x \in W_{n}, x \neq x_{m}, y_{m}$ предел (8) сушествует, и, таким образом, нужно установить только, что предел (8) существует при $x=x_{m}, y_{m}$.

Для путей $\pi_{1}, \pi_{2}$ всегда имеется такое число $m_{1} \geqslant 0$, что $x_{i}=y_{i}$, если $i=\overline{0, m_{1}}$, и $x_{i} \neq y_{i}$, если $i>m_{1}$.

Пусть $1 \leqslant m \leqslant n$. Если рассмотреть две совокупности $\left\{\tilde{h}_{x}^{(n)}, x \in V_{n}\right\}$ и $\left\{\hat{h}_{x}^{(n)}\right.$, $\left.x \in V_{n}\right\}$, отвечающие двум значениям $\tilde{h}_{x}^{(n)}$ и $\hat{h}_{x}^{(n)}$ при $x=x_{n}, y_{n}$ в (7), то из равенства (4) и неравенства $\left|f_{h}^{\prime}(h ; \theta)\right| \leqslant \theta$ следует, что

$$
\left|\hat{h}_{x_{m-1}}^{(n)}-\tilde{h}_{x_{m-1}}^{(n)}\right| \leqslant\left\{\begin{array}{l}
\left|\theta_{x_{m-1} x_{m}}\right|\left|\hat{h}_{x_{m}}^{(n)}-\tilde{h}_{x_{m}}^{(n)}\right|, \text { если } m<m_{1} \text { или } m>m_{1}, \\
\left|\theta_{x_{m-1} x_{m}}\right|\left|\hat{h}_{x_{m}}^{(n)}-\tilde{h}_{x_{m}}^{(n)}\right|+\left|\theta_{y_{m-1} y_{m}}\right|\left|\hat{h}_{y_{m}}^{(n)}-\tilde{h}_{y_{m}}^{(n)}\right|, \text { если } m=m_{1},
\end{array}\right.
$$


И

$$
\left|\hat{h}_{y_{m-1}}^{(n)}-\tilde{h}_{y_{m-1}}^{(n)}\right| \leqslant\left\{\begin{array}{l}
\left|\theta_{y_{m-1} y_{m}}\right|\left|\hat{h}_{y_{m}}^{(n)}-\tilde{h}_{y_{m}}^{(n)}\right|, \text { если } m<m_{1} \text { или } m>m_{1}, \\
\left|\theta_{x_{m-1} x_{m}}\right|\left|\hat{h}_{x_{m}}^{(n)}-\tilde{h}_{x_{m}}^{(n)}\right|+\left|\theta_{y_{m-1} y_{m}}\right|\left|\hat{h}_{y_{m}}^{(n)}-\tilde{h}_{y_{m}}^{(n)}\right|, \text { если } m=m_{1} .
\end{array}\right.
$$

Итерируя эти оценки, получаем

$$
\begin{aligned}
& \left|\hat{h}_{x_{m}}^{(n)}-\tilde{h}_{x_{m}}^{(n)}\right| \leqslant \\
& \leqslant\left\{\begin{array}{l}
\prod_{i=1}^{n-m}\left|\theta_{x_{m-1+i} x_{m+i}}\right|\left|\hat{h}_{x_{n}}^{(n)}-\tilde{h}_{x_{n}}^{(n)}\right|, \text { если } m<n<m_{1} \text { или } m_{1}<m<n \\
\prod_{i=1}^{n-m}\left|\theta_{x_{m-1+i} x_{m+i}}\right|\left|\hat{h}_{x_{n}}^{(n)}-\tilde{h}_{x_{n}}^{(n)}\right|+ \\
\quad+\prod_{i=1}^{n-m}\left|\theta_{y_{m-1+i} y_{m+i}}\right|\left|\hat{h}_{y_{n}}^{(n)}-\tilde{h}_{y_{n}}^{(n)}\right|, \text { если } m \leqslant m_{1}<n .
\end{array}\right.
\end{aligned}
$$

Аналогично имеем

$$
\begin{aligned}
& \left|\hat{h}_{y_{m}}^{(n)}-\tilde{h}_{y_{m}}^{(n)}\right| \leqslant \\
& \leqslant\left\{\begin{array}{l}
\prod_{i=1}^{n-m}\left|\theta_{y_{m-1+i} y_{m+i}}\right|\left|\hat{h}_{y_{n}}^{(n)}-\tilde{h}_{y_{n}}^{(n)}\right|, \text { если } m<n<m_{1} \text { или } m_{1}<m<n, \\
\prod_{i=1}^{n-m}\left|\theta_{x_{m-1+i} x_{m+i}}\right|\left|\hat{h}_{x_{n}}^{(n)}-\tilde{h}_{x_{n}}^{(n)}\right|+ \\
\quad+\prod_{i=1}^{n-m}\left|\theta_{y_{m-1+i} y_{m+i}}\right|\left|\hat{h}_{y_{n}}^{(n)}-\tilde{h}_{y_{n}}^{(n)}\right|, \text { если } m \leqslant m_{1}<n .
\end{array}\right.
\end{aligned}
$$

В силу условия I $\theta_{x y}=\theta_{i j}$, если $x \in H_{i}, y \in H_{j}, i, j=\overline{1, r-1}$. Введем обозначение $\theta=\max \left\{\left|\theta_{i j}\right| ; i, j=\overline{1, r-1}\right\}$.

Пусть теперь даны произвольные $N, M>n$ и совокупности $\left\{h_{x}^{(N)}, x \in V_{N}\right\}$ и $\left\{h_{x}^{(M)}\right.$, $\left.x \in V_{M}\right\}$, определяемые начальными условиями вида (6) при $x \in W_{N}$ и $x \in W_{M}$, соответственно, и рекуррентными уравнениями (4). Положим $\tilde{h}_{x}^{(n)}=h_{x}^{(N)}, \hat{h}_{x}^{(n)}=h_{x}^{(M)}$ при $x=x_{n}, y_{n}$. Тогда из неравенства (9) имеем

$$
\left|h_{x_{m}}^{(N)}-h_{x_{m}}^{(M)}\right| \leqslant 4 C \theta^{n-m}, \quad\left|h_{y_{m}}^{(N)}-h_{y_{m}}^{(M)}\right| \leqslant 4 C \theta^{n-m},
$$

где

$$
C=k \max _{h}|f(h ; \theta)|=k \operatorname{arcth} \theta .
$$

Из этой оценки вытекает, что последовательности $h_{x_{m}}^{(n)}$ и $h_{y_{m}}^{(n)}$ удовлетворяют при $n \rightarrow \infty$ и фиксированном $m$ критерию Коши и, следовательно, предел (8) сушествует и не зависит от выбора функций $h_{x_{n}}^{(n)}$ и $h_{y_{n}}^{(n)}$ в $(7)$. Поскольку допредельные совокупности $\left\{h_{x}^{(n)}\right\}$ удовлетворяют по построению уравнениям (4), то и $\left\{h_{x}\right\}$ удовлетворяет этим уравнениям. Единственность $\left\{h_{x}\right\}$ следует, очевидно, из оценки (9). Теорема доказана. 
Лемма 1. Для любого $t, s \in Q_{k}, t \leqslant s$,

$$
t=\sum_{n=1}^{N_{1}} \frac{i_{n}}{k^{n}}, \quad s=\sum_{n=1}^{N_{2}} \frac{j_{n}}{k^{n}},
$$

совпадают следующие совокупности чисел:

a) $\mathbf{h}^{\pi_{1}(t, 1) \pi_{2}(s)} u \mathbf{h}^{\pi_{1}(t, 2) \pi_{2}(s)} ; h_{x}^{\pi_{1}(t, 1) \pi_{2}(s)}=0$, если $x=x_{n}, \quad n \geqslant N_{1}+1$;

б) $\mathbf{h}^{\pi_{1}(t) \pi_{2}(s, 1)} u \mathbf{h}^{\pi_{1}(t) \pi_{2}(s, 2)} ; \quad h_{x}^{\pi_{1}(t) \pi_{2}(s, 1)}=h_{i}^{*}$, ecлu $x=y_{n} \in H_{i}, \quad i=\overline{0, r-1}$, $n \geqslant N_{2}+1$.

ДокАЗАТЕЛЬСТво. Случай “а”. Пусть $\pi_{1}=\pi_{1}(t, 1)$. Положим при $n>N_{1}$

$$
h_{x}= \begin{cases}-h_{i}^{*}, & \text { если } x \prec x_{n} \text { и } x \in H_{i}, \quad i=\overline{0, r-1}, \\ 0, & \text { если } x_{n} \preceq x \prec y_{n}, \\ h_{i}^{*}, & \text { если } y_{n} \preceq x \text { и } x \in H_{i}, \quad i=\overline{0, r-1},\end{cases}
$$

и доопределим совокупность $\left\{h_{x}, x \in V\right\}$ при $x \in V_{N_{1}}$, пользуясь рекуррентньми уравнениями (4). Тогда совокупность $\left\{h_{x}, x \in V\right\}$, как легко видеть, удовлетворяет этим уравнениям при всех $x \in V$, и вследствие условия единственности в теореме 3 построенная совокупность $\left\{h_{x}, x \in V\right\}$ и есть $\left\{h_{x}^{\pi_{1}(t, 1) \pi_{2}(s)}, x \in V\right\}$. По тем же самым соображениям совокупность $\left\{h_{x}, x \in V\right\}$ совпадает и с $\left\{h_{x}^{\pi_{1}(t, 2) \pi_{2}(s)}, x \in V\right\}$, следовательно,

$$
\mathbf{h}^{\pi_{1}(t, 1) \pi_{2}(s)}=\mathbf{h}^{\pi_{1}(t, 2) \pi_{2}(s)} .
$$

Аналогично доказывается случай “б”. Лемма доказана.

Из этой леммы следует, что для любой точки $(t ; s) \in D=\left\{\left(t_{1}, t_{2}\right) \in[0 ; 1+1 / k] \times\right.$ $\left.[0 ; 1+1 / k]: t_{1} \leqslant t_{2}\right\}$ однозначно определяется совокупность чисел $\mathbf{h}^{\pi_{1}(t) \pi_{2}(s)}$.

Пусть $h_{0}(t, s)=h_{x^{0}}^{\pi_{1}(t) \pi_{2}(s)}$, где $x^{0}-$ граничная вершина графа $\Gamma^{k}$.

ЛЕмма 2. Функция $h_{0}(t, s),(t, s) \in D$, является строго убьвающей непрерывной функиией относительно каждой из переменных $t, s$ и $h_{0}(0,0)=h_{0}^{*}, h_{0}(0,1+$ $1 / k)=0, \quad h_{0}(1+1 / k, 1+1 / k)=-h_{0}^{*}$.

ДокАЗАТЕЛЬСТво. Пусть

$$
t_{1}=\sum_{n=1}^{\infty} \frac{i_{n}}{k^{n}}, \quad t_{2}=\sum_{n=1}^{\infty} \frac{j_{n}}{k^{n}}
$$

и $t_{1}>t_{2}$. Тогда сушествует такое $N$, что

$$
k^{N} \sum_{n=1}^{N} \frac{i_{n}}{k^{n}}-k^{N} \sum_{n=1}^{N} \frac{j_{n}}{k^{n}} \geqslant 2 .
$$


Пусть $\pi_{1}\left(t_{1}\right)=\left\{x^{0}=x_{0}^{(1)}<x_{2}^{(1)}<\cdots\right\}$ и $\pi_{1}\left(t_{2}\right)=\left\{x^{0}=x_{0}^{(2)}<x_{2}^{(2)}<\cdots\right\}$. Тогда из (10) следует, что $x_{N}^{(2)} \prec x_{N}^{(1)}$ и между $x_{N}^{(2)}, x_{N}^{(1)}$ сушествует еше по меньшей мере одна вершина $x_{N}$, т.е. $x_{N}^{(2)} \prec x_{N} \prec x_{N}^{(1)}$. По условиям (6) отсюда следует, что

$$
\begin{aligned}
& h_{x}^{\pi_{1}\left(t_{1}\right) \pi_{2}} \leqslant h_{x}^{\pi_{1}\left(t_{2}\right) \pi_{2}}, \quad x \in W_{N} ; \\
& h_{x}^{\pi_{1}\left(t_{1}\right) \pi_{2}}=-h_{i}^{*}<h_{x}^{\pi_{1}\left(t_{2}\right) \pi_{2}}=0, \text { если } x=x_{N} \in H_{i}, \quad i=\overline{0, r-1} .
\end{aligned}
$$

Тогда с помошью (4) из (11) для любого $n \leqslant N$ имеем

$$
\begin{array}{ll}
h_{x}^{\pi_{1}\left(t_{1}\right) \pi_{2}} \leqslant h_{x}^{\pi_{1}\left(t_{2}\right) \pi_{2}}, & x \in W_{n} ; \\
h_{x}^{\pi_{1}\left(t_{1}\right) \pi_{2}}<h_{x}^{\pi_{1}\left(t_{2}\right) \pi_{2}}, & \text { если } x=x_{n} .
\end{array}
$$

Полагая $n=0$, мы получаем, что $h_{x^{0}}^{\pi_{1}\left(t_{1}\right) \pi_{2}}<h_{x^{0}}^{\pi_{1}\left(t_{2}\right) \pi_{2}}$, это доказывает строгую монотонность функции $h_{0}(t, s)$ относительно $t$. Докажем ее непрерывность (относительно $t$ ). Пусть функции $\pi_{1}\left(t_{1}\right)$ и $\pi_{2}\left(t_{2}\right)$ до уровня $N$ совпадают. Положим $\tilde{h}_{x_{N}}^{(N)}=h_{x_{N}}^{\pi_{1}\left(t_{1}\right) \pi_{2}}$ и $\hat{h}_{x_{N}}^{(N)}=h_{x_{N}}^{\pi_{1}\left(t_{2}\right) \pi_{2}}$. Тогда очевидно, что $\left|h_{0}\left(t_{1}, s\right)-h_{0}\left(t_{2}, s\right)\right| \leqslant 4 C \theta^{N}$, если $\mid t_{1}-$ $t_{2} \mid \leqslant k^{-N-1}$. Из этой оценки следует, что

$$
\left|h_{0}\left(t_{1}, s\right)-h_{0}\left(t_{2}, s\right)\right| \leqslant C_{1}\left|t_{1}-t_{2}\right|^{\alpha}, \quad \alpha=-\frac{\log \theta}{\log k},
$$

это доказывает непрерывность функции $h_{0}(t, s)$ относительно $t$. Аналогично проводится доказательство относительно $s$.

Соотношения $h_{0}(0,0)=h_{0}^{*}, h_{0}(0,1+1 / k)=0, h_{0}(1+1 / k, 1+1 / k)=-h_{0}^{*}$ следуют из леммы 1. Лемма доказана.

Из леммы 2 следует, что совокупности чисел $\mathbf{h}^{\pi_{1}(t) \pi_{2}(s)}$ различны при различных $(t, s) \in D$.

Обозначим через $\mu(t, s)$ гиббсовскую меру, соответствующую совокупности величин $\mathbf{h}^{\pi_{1}(t) \pi_{2}(s)},(t, s) \in D$. Из вьшеизложенного вытекает основной результат данной статьи.

ТЕОРема 4. Для любой точки $(t, s) \in D$ существует единственная гиббсовская мера $\mu(t, s)$, и при этом

$$
\mu(0,0)=\mu_{2}, \quad \mu\left(0,1+\frac{1}{k}\right)=\mu_{1}, \quad \mu\left(1+\frac{1}{k}, 1+\frac{1}{k}\right)=\mu_{0}
$$

Поскольку распределения $\mu(t, s)$ различны при различных $(t, s) \in D$, мы получаем континуум предельных гиббсовских мер.

Благодарности. В заключение автор хотел бы выразить свою признательность Н. Н. Ганиходжаеву за внимание к работе. 


\section{Список литературы}

[1] У. А. Розиков. ТМф. 1997. Т. 112. № 1. С. 170-175.

[2] Н. Н. Ганиходжсаев. ДАН РУз. 1994. № 5. С. 3-6.

[3] Н. Н. Ганиходжаев, У. А. Розиков. ТМФ. 1997. Т. 111. № 1. С. 109-117.

[4] P. M. Bleher. Commun. Math. Phys. 1990. V. 128. P. 411-419.

[5] S. Katsura, M. Takizawa. Progr. Theor. Phys. 1974. V. 51. P. 82-98.

[6] Р. Л. Добрушин. Теория вероятн. и ее примен. 1973. Т. 18. В. 2. С. 261-279.

[7] П. М. Блехер, Н. Н. Ганиходжаев. Теория вероятн. и ее примен. 1990. Т. 35. В. 2. C. $220-230$.

[8] Н. Н. Ганиходжаев, У.А. Розиков. УзМЖ. 1995. № 4. С. 8-19.

[9] Н. Н. Ганиходжаев. ТМФ. 1990. Т. 85. № 2. С. 163-175.

[10] К. Престон. Гиббсовские состояния на счетных множествах. М.: Мир, 1977.

[11] F. Spitzer. Ann. Prob. 1975. V. 3. P. 387-398.

Поступила в редакцию 11.III.1998 г., после доработки 4.VI.1998 г. 